\title{
Evaluación del Plan de Manejo Ambiental para una Planta de Producción de Biodiesel
}

\section{Evaluation of Enviromental Management Planing for a Biodiesel Production Plant}

\author{
Maldonado Romero María del Mar', Torres Ortega Jesús Alfonso² \\ ${ }^{1}$ Facultad de Ingeniería, Programa de Ingeniería Ambiental y Sanitaria, Universidad de La Salle, Bogotá, Colombia, \\ marimara@hotmail.com \\ 2 Facultad de Ingeniería, Programa de Ingeniería Ambiental y Sanitaria, Universidad de La Salle, Bogotá, Colombia, \\ jatorres@unisalle.edu.co
}

\section{Resumen}

Aceites Manuelita S. A. es una empresa dedicada al cultivo de palma de aceites a la producción y comercialización de Biodiesel, actualmente la empresa procesa el fruto de sus cultivos propios de 14.000 hectáreas.

Aceites Manuelita S. A., es una empresa en crecimiento; debido a este y a la incursión de una nueva línea de producción con un nuevo producto, se hizo necesaria la realización de un Plan de Manejo Ambientall. En 2008, fue adaptado a las necesidades de la empresa, en ese entonces y en las proyecciones con que se contaba; hoy, las cosas han cambiado y lo que se había estimado ya no es aplicable a la actual, lo que hace necesaria una evaluación del documento actual y el replanteamiento del mismo, haciendo especial énfasis en la gestión de los residuos que se generan y en los impactos producidos por la actividad. Como campo de acción para el desarrollo del proyecto se propone abarcar los residuos de tipo industrial, generados en el normal desarrollo de las actividades de la empresa.

Mediante una combinación de metodologías que integran a la comunidad con el uso de mejores tecnologías, se logró el manejo de los residuos y los subproductos industriales que se generan, se evaluó y complementó el Plan de Manejo Ambiental existente, planteando nuevas alternativas da largo plazo para el manejo de los residuos y subproductos industriales de la empresa. Todo esto con el fin de lograr unas instalaciones adecuadas para su manejo, acopio temporal y tratamiento de los mismos, también para una mayor conciencia de la comunidad en cuanto a la protección ambiental y al aporte que cada uno hace a diario desde cada una de sus actividades.

Palabras Clave: impacto, biodiesel, residuo, subproducto, aceite, palma.

\section{Abstract}

Manuelita Oils SA is a company dedicated to the cultivation of palm, production and marketing of biodiesel, the company currently processes the fruit of their own crops. Currently Manuelita Oils SA is a growing company. Because the incursion of a new production line with a new product was necessary to does an environmental management design, in 2008, this was adapted to the needs of the company at the time and in the projections that were available, today, things have changed and what had been estimated at that time is no longer applicable to current realities, requiring an assessment of the current document and the rethinking of the same, with special emphasis in the management of waste generated and the impacts of the activity. As a field of action for the development of the project is intended to cover industrial waste type, which are generated in the normal course of business activities. Through a combination of methodologies that integrate the community with the use of better technologies achieved the management of waste and industrial byproducts that are generated by assessing and complementing the existing an environmental management design, raising new longterm options for handling waste and industrial products company and not only achieving adequate 
facilities for handling, temporary storage and processing data but also greater community awareness about environmental protection and the contribution that each makes daily from each of their activities.

Keywords: impact, biodiesel, waste, by-product, oil, palm.

\section{Introducción}

Este documento contiene las directrices poa seguir para que las actividades desarrolladas en una planta de producción de biodiesel se adelanten de manera sustentable y con el mínimo deterioro ambiental posible. Además, contempla todos los aspectos que requieren las autoridades ambientales competentes para este tipo de procesos.

Aceites Manuelita S.A., con el objetivo de que las actividades que realiza se adelanten bajo consideraciones ambientales adecuadas y conscientes de acatar la legislación ambiental vigente, cuenta con un Plan de Manejo Ambiental (PMA) para las actividades de plantación de palma africana, producción de aceite de palma y producción de biodiesel, en sus instalaciones ubicadas en el Municipio de San Carlos de Guaroa, Departamento del Meta [1].

En el PMA, se identifican los impactos ambientales que generan las actividades que realiza Aceites Manuelita S.A., en su área de influencia y propone las medidas para mitigar, controlar y compensar dichos impactos [2], [3], [4]. Los procesos de producción en Aceites Manuelita S.A., comprenden dos grandes fases: la agrícola, compuesta por la plantación de palma de aceite y la industrial, compuesta por la operación de la Planta Extractora de Yaguarito y Palmar de Manavire y la Planta de Producción de Biodiesel. Para la plantación de palma de aceite, Aceites Manuelita S. A., cuenta en primera instancia con una extensión de 6135 hectáreas en los predios de la hacienda Yaguarito - Inspección de Surimena, Municipio de San Carlos de Guaro; y, por otro lado, con 1967,5 hectáreas en los predios de Palmar de Manavire, la cual se encuentra en la Vereda Pesqueros - Inspección de la Palmera, Municipio de San Carlos de Guaroa.

Igualmente, cuenta con dos plantas extractoras: la primera se encuentra en la hacienda Yaguarito, con una capacidad producción de 55 toneladas de RFF/hora (RFF: Racimos de Fruto Fresco), proyectada para expandirse hasta 70 toneladas de RFF/hora. La segunda se denomina Palmar de Manavire, en la Vereda Pesqueros, con una capacidad de procesamiento de 17 toneladas de RFF/hora. Anualmente, estas dos plantas extractoras producen un total aproximado de 80.000 toneladas anuales de aceite crudo de palma.

La gestión ambiental en Aceites Manuelita S. A. (AMSA), se manejaba por medio de la aplicación de los programas estipulados en el Plan de Manejo Ambiental (PMA), establecido para la plantación y planta extractora, aprobado por CORPORINOQUIA mediante Resolución 473 del 11 de Agosto de 2000, y el cumplimiento de requisitos legales y autorizaciones ambientales para el uso de recursos exigidos por las autoridades ambientales competentes.

Dentro del mejoramiento de la gestión ambiental empresarial - AMSA, se encuentra actualmente en el proceso de certificación de la norma ISO 14001, para lo cual se ha generado un cronograma que establece una serie de metas por cumplir. Dentro de estas se encuentran como principales la identificación de los aspectos e impactos ambientales y la evaluación de la conformidad con los requisitos legales en la generación de la política, objetivos y metas ambientales hacia los procesos y actividades empresariales [5].

El presente documento, conforma una de las metas trazadas en el proceso de certificación de la norma ISO 14000 y como cumplimiento de las regulaciones ambientales establecidas por las autoridades ambientales; además, de convertirse en una herramienta importante en la generación y establecimiento de los objetivos, metas, planes y programas ambientales que la empresa desarrollará en la creación del Sistema de Gestión Ambiental (SGA), esto por medio de la aplicación y seguimiento de las fichas de manejo ambiental propuestas en el presente documento [5]. Es así como, este documento servirá para tenerse en cuenta al momento de evaluar y reformular el PMA para el conjunto de una plantación de palma africana, planta extractora de aceite y operación de una planta productora de biodiesel [6]. 


\section{Descripción del Proceso}

Después de la recolección y almacenamiento del fruto, esta pasa a un proceso de esterilización, luego se retira el fruto de su soporte natural para llevarlo a los digestores. La función del digestor es acondicionar el fruto, haciendo que se separe el mesocarpio de las nueces para facilitar en el prensado la extracción del aceite. El aceite extraído es recolectado, allí le son retiradas parte de las impurezas para el proceso tales como fibras, arena, etc. y luego es bombeada a la sección de clarificación (ver Figura 1).

Proceso de refinación del aceite: la refinación del aceite crudo de palma busca la eliminación de compuestos indeseables para la producción de biodiesel. El proceso inicia con la adición de ácido cítrico y tierras de blanqueo que capturan estos compuestos, los cuales posteriormente, mediante un filtrado, son removidos del aceite. A continuación el aceite se somete a condiciones de vacío y alta temperatura, lo que permito así la evaporación y remoción de los ácidos grasos libres, dando como producto final el aceite refinado.

Proceso de producción de biodiesel: la producción de biodiesel se realiza por medio de una reacción química del aceite refinado (RBD: Refinado, blanqueado y desodorizado) con metanol en presencia de hidróxido de sodio, formando una fase liviana de metilester (biodiesel) y una fase pesada de glicerina cruda (ver Figura 2). El biodiesel es sometido a un lavado con ácido cítrico para su neutralización y posterior centrifugación, esto permito la remoción de otras impurezas (sales), posteriormente, el biodiesel es calentado para reducir las trazas de humedad y, finalmente, enfriado para su almacenamiento final.

Proceso de purificación de la glicerina: la glicerina cruda obtenida durante la formación del biodiesel es sometida a un proceso de acidificación para la remoción de jabones, luego se neutraliza formando salmuera. Posteriormente, la glicerina cruda es sometida a un proceso de destilación en una columna a condiciones de vacío y alta temperatura, evaporando solamente la glicerina la cual se purifica al ir eliminando la sal presente, para luego ser filtrada con carbón activado obteniendo de esta manera la glicerina refinada.

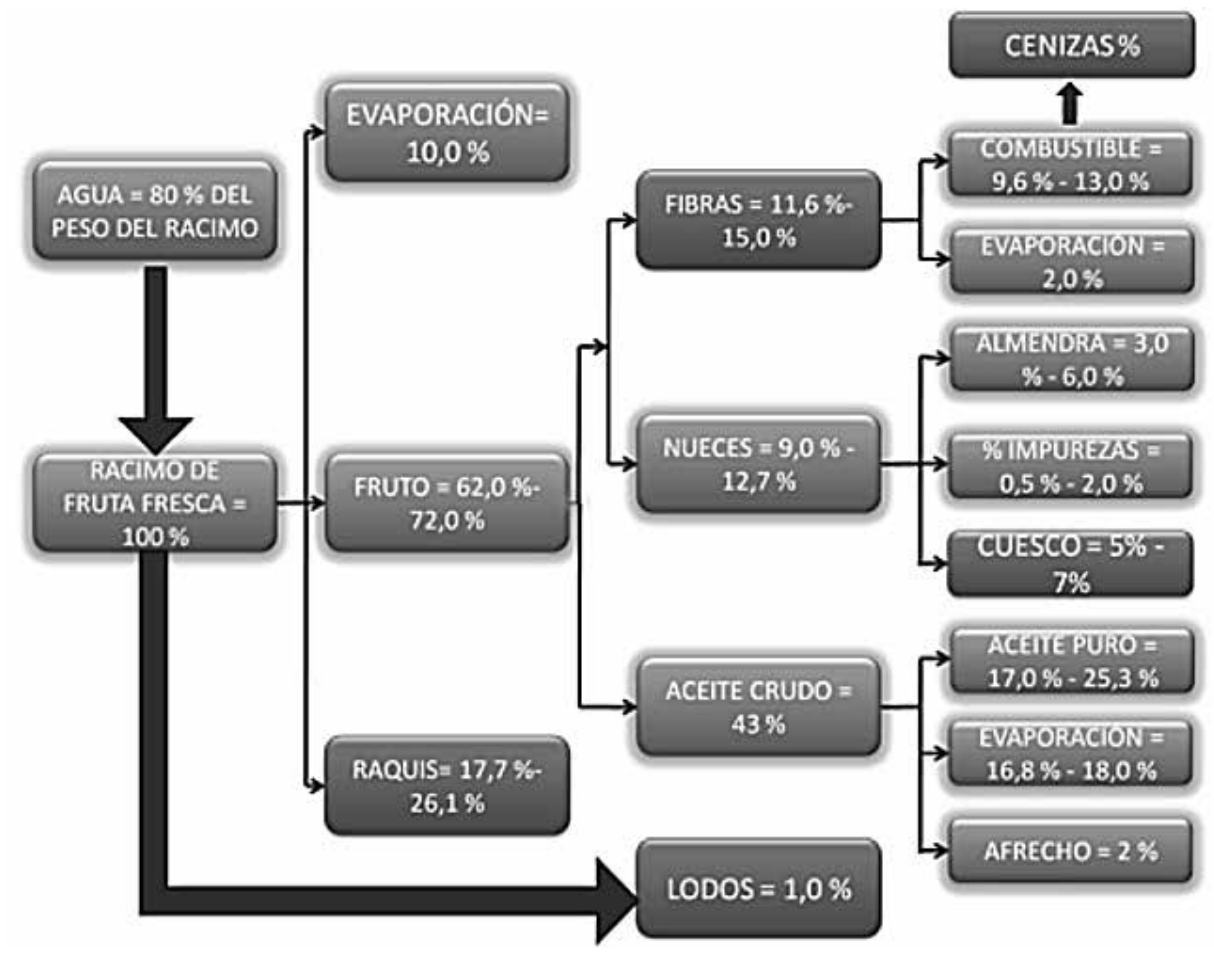

Figura 1. Componentes del racimo de palma de aceite. 


\section{Planteamiento de Alternativas}

Para el diseño de un PMA en particular se debe centras en los aspectos e impactos ambientales así como en los residuos y subproductos industriales [7], [8], [9].

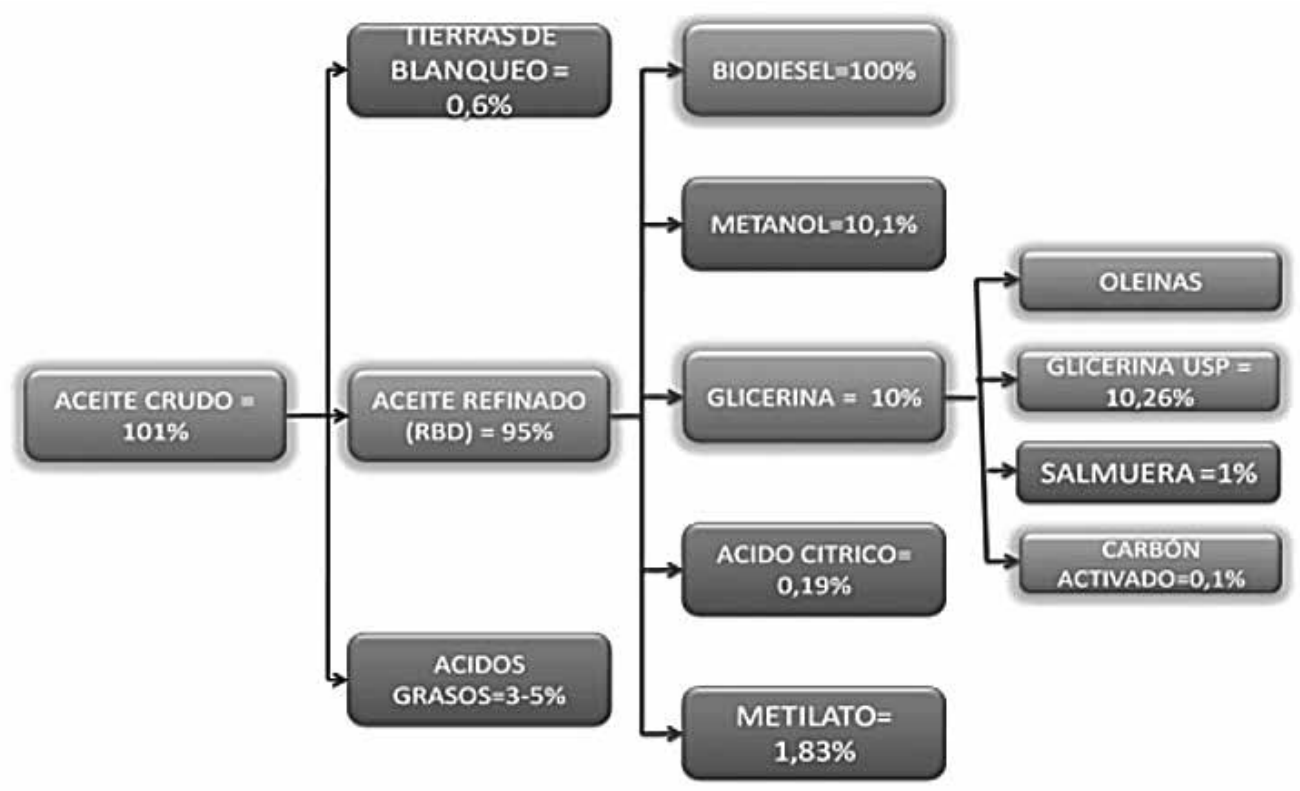

Fuente: (Arenas, 2010; Conesa, 2009; Infante, 2007).

Figura 2. Composiciones de los productos obtenidos en la fabricación de biodiesel a partir de aceite de palma.

\section{Matriz DOFA}

Para realizar el planteamiento de las estrategias de control de impactos ambientales, se hace uso del análisis Debilidades, Oportunidades, Fortalezas, Amenazas (DOFA); mediante el planteamiento de planes y proyectos que permitan el control de los factores internos y externos ens el manejo de residuos sólidos y subproductos industriales [10].

El desarrollo de la herramienta DOFA tiene como base la revisión documental inicial y en las visitas de campo realizadas durante la fase de diagnóstico y de participación de la comunidad. Se identifican los factores internos (Fortalezas y Debilidades) los cuales de forma directa pueden ser mejorados y los factores externos (Oportunidades y Amenazas) los cuales son dependientes a ser modificados 0 mitigados. Una vez establecidas, se construyen las estrategias a partir de las siguientes relaciones:
FO (Fortalezas-Oportunidades
: maximizar / maximizar
DO (Debilidades- Oportunidades
: minimizar / maximizar
FA (Fortalezas-Amenazas
: maximizar / minimizar
DA (Debilidades-Amenazas
: minimizar / minimizar

Finalmente, se crean los planes y para el desarrollo de estrategias y proponen metas a corto, medio y largo plazo con el fin de minimizar y controlar los impactos asociados a los residuos sólidos y subproductos industriales como se describe en la siguiente Tabla 1. 
Tabla 1. Matriz DOFA [11].

\begin{tabular}{|c|c|c|c|c|c|}
\hline \multicolumn{6}{|c|}{ DEBILIDADES } \\
\hline D1 & $\begin{array}{l}\text { No hay programas de edu- } \\
\text { cación ni campañas de } \\
\text { separación en la fuente. }\end{array}$ & D2 & \begin{tabular}{|l|} 
Falta de presupuesto para la \\
implementación de programas \\
y proyectos ambientales.
\end{tabular} & D3 & $\begin{array}{l}\text { No se tienen implementadas } \\
\text { rutas para la recolección de } \\
\text { residuos aprovechables. }\end{array}$ \\
\hline D4 & $\begin{array}{l}\text { Desconocimiento del po- } \\
\text { tencial aprovechamiento } \\
\text { de los residuos sólidos por } \\
\text { parte de la comunidad. }\end{array}$ & D5 & $\begin{array}{l}\text { La empresa cuenta con po- } \\
\text { blación flotante, lo que genera } \\
\text { que esta comunidad no se } \\
\text { entere de los programas y no } \\
\text { genere sentido de pertenencia. }\end{array}$ & D6 & $\begin{array}{l}\text { Se tiene un procedimiento } \\
\text { detallado para la recolección } \\
\text { de residuos pero no se ha } \\
\text { difundido ni implementado. }\end{array}$ \\
\hline D7 & $\begin{array}{l}\text { No existe diagramación de } \\
\text { rutas para recolección. }\end{array}$ & D8 & $\begin{array}{l}\text { No se cuenta con un grupo } \\
\text { de recicladores, ya que no se } \\
\text { tiene conciencia de su necesi- } \\
\text { dad. }\end{array}$ & D9 & $\begin{array}{l}\text { No se realizan los pesajes de } \\
\text { los residuos generados, por } \\
\text { lo tanto no se tienen datos } \\
\text { permanentes de los mismos. }\end{array}$ \\
\hline D10 & $\begin{array}{l}\text { No hay control de las can- } \\
\text { tidades de residuos que se } \\
\text { generan. }\end{array}$ & D11 & $\begin{array}{l}\text { No se cuenta con un centro de } \\
\text { acopio de los residuos peligro- } \\
\text { sos. }\end{array}$ & D12 & $\begin{array}{l}\text { La ubicación de la empresa } \\
\text { no permite hacer una re- } \\
\text { colección ni un manejo de los } \\
\text { residuos de forma constante. }\end{array}$ \\
\hline D13 & $\begin{array}{l}\text { No se ha implementado el } \\
\text { Plan de Gestión Integral de } \\
\text { Residuos Sólidos. }\end{array}$ & D14 & $\begin{array}{l}\text { La planta no cuenta con un } \\
\text { lugar específico para el manejo } \\
\text { y la disposición de residuos y } \\
\text { subproductos }\end{array}$ & D15 & $\begin{array}{l}\text { No se cuenta con actividades } \\
\text { de comercialización de los } \\
\text { residuos. }\end{array}$ \\
\hline D16 & $\begin{array}{l}\text { Falta de personal capacita- } \\
\text { do (operario no calificado) }\end{array}$ & D17 & $\begin{array}{l}\text { El inadecuado manejo de los } \\
\text { residuos sólidos en algunos } \\
\text { sectores, estos son dispuestos } \\
\text { en el suelo directamente, incre- } \\
\text { mentando su contaminación. }\end{array}$ & & \\
\hline \multicolumn{2}{|r|}{ FORTALEZAS } & \multicolumn{2}{|r|}{ OPORTUNIDADES } & \multicolumn{2}{|r|}{ AMENAZAS } \\
\hline $\mathrm{F} 1$ & $\begin{array}{l}\text { Se cuenta con la dotación } \\
\text { de elementos para la sepa- } \\
\text { ración en la fuente. }\end{array}$ & O1 & $\begin{array}{l}\text { La empresa puede llegar a uti- } \\
\text { lizar y aprovechar algunos de } \\
\text { los residuos. }\end{array}$ & A1 & $\begin{array}{l}\text { Pérdidas económicas por el } \\
\text { no aprovechamiento de re- } \\
\text { siduos sólidos reciclables. } \\
\end{array}$ \\
\hline $\mathrm{F} 2$ & $\begin{array}{l}\text { Se cuenta con la volun- } \\
\text { tad de los directivos de la } \\
\text { empresa para mejorar la } \\
\text { gestión de los residuos. }\end{array}$ & $\mathrm{O} 2$ & $\begin{array}{l}\text { El manejo de los residuos pu- } \\
\text { ede ser mejorado con nuevas y } \\
\text { mejores tecnologías. }\end{array}$ & $\mathrm{A} 2$ & $\begin{array}{l}\text { Incremento en la generación } \\
\text { de residuos por el incremento } \\
\text { en la población. }\end{array}$ \\
\hline F3 & $\begin{array}{l}\text { El manejo externo de resid- } \\
\text { uos hospitalarios se realiza } \\
\text { con un gestor autorizado. }\end{array}$ & O3 & $\begin{array}{l}\text { Posibilidad de construir y } \\
\text { operar una planta de aprove- } \\
\text { chamiento de residuos sólidos. }\end{array}$ & A3 & $\begin{array}{l}\text { Contaminación de los recur- } \\
\text { sos naturales por técnicas de } \\
\text { disposición ambiental inse- } \\
\text { guras. }\end{array}$ \\
\hline $\mathrm{F} 4$ & $\begin{array}{l}\text { Algunos tipos de residuos } \\
\text { cuentan con gestores para } \\
\text { su disposición. }\end{array}$ & $\mathrm{O} 4$ & $\begin{array}{l}\text { Capacitar al personal para el } \\
\text { manejo y aprovechamiento de } \\
\text { los residuos. }\end{array}$ & A4 & $\begin{array}{l}\text { Dificultad en el transporte y } \\
\text { posibilidad de no disponer los } \\
\text { residuos. }\end{array}$ \\
\hline F5 & $\begin{array}{l}\text { Se cuenta con un camión } \\
\text { con la capacidad de trans- } \\
\text { portar los residuos dos } \\
\text { veces a la semana. }\end{array}$ & $\mathrm{O5}$ & $\begin{array}{l}\text { Realizar prácticas de seg- } \\
\text { regación en la fuente. }\end{array}$ & A5 & $\begin{array}{l}\text { No siempre se cumplen los } \\
\text { tiempos de recolección. }\end{array}$ \\
\hline F6 & $\begin{array}{l}\text { Se está realizando la ad- } \\
\text { ecuación al área de acopio } \\
\text { de los residuos aprovech- } \\
\text { ables. }\end{array}$ & & & & \\
\hline
\end{tabular}




\section{Estrategias para el manejo y disposición de los residuos}

A partir de la matriz DOFA se procede a plantear las alternativas para el correcto manejo y disposición de los residuos como se muestra en la Tabla 2.

Tabla 2. Evaluación de Alternativas [11].

\begin{tabular}{|c|c|c|c|}
\hline \multicolumn{2}{|r|}{$\begin{array}{l}\text { FORTALEZAS - OPORTUNIDADES } \\
\text { (MAX:MAX) }\end{array}$} & \multicolumn{2}{|r|}{ FORTALEZAS - AMENAZAS (MAX:MIN) } \\
\hline F01 & $\begin{array}{l}\text { Fortalecimiento de la gestión ambiental en } \\
\text { la empresa. }\end{array}$ & FA1 & $\begin{array}{l}\text { Evitar prácticas de disposición final de re- } \\
\text { siduos sólidos ambientalmente inseguras, } \\
\text { mediante el fortalecimiento de recolección y } \\
\text { transporte de residuos. }\end{array}$ \\
\hline FO2 & $\begin{array}{l}\text { Sensibilización, educación y participación } \\
\text { de la comunidad. }\end{array}$ & FA2 & $\begin{array}{l}\text { Capacitación a los integrantes de la cadena } \\
\text { de gestión de residuos sólidos. }\end{array}$ \\
\hline FO3 & $\begin{array}{l}\text { Aprovechar los residuos mediante la com- } \\
\text { ercialización. }\end{array}$ & FA3 & $\begin{array}{l}\text { Fortalecimiento de instrumentos de plani- } \\
\text { ficación, organizacionales, normativos y } \\
\text { financieros, relacionados con la gestión inte- } \\
\text { gral de los residuos sólidos. }\end{array}$ \\
\hline FO4 & $\begin{array}{l}\text { Análisis y evaluación de tecnologías ap- } \\
\text { ropiadas para la recuperación y aprove- } \\
\text { chamiento. }\end{array}$ & FA4 & $\begin{array}{l}\text { Ajuste de procesos operativos de recolec- } \\
\text { ción y transporte a los centros de acopio. }\end{array}$ \\
\hline \multirow[t]{2}{*}{ FO5 } & $\begin{array}{l}\text { Recuperación, aprovechamiento y comer- } \\
\text { cialización de los residuos. }\end{array}$ & FA5 & $\begin{array}{l}\text { Mejorar los sistemas de eliminación, trata- } \\
\text { miento y disposición final de los residuos. }\end{array}$ \\
\hline & & FA6 & $\begin{array}{l}\text { Fortalecimiento en la gestión ambiental de } \\
\text { la empresa mediante la optimización del } \\
\text { manejo de residuos sólidos. }\end{array}$ \\
\hline \multicolumn{2}{|c|}{ DEBILIDADES - OPORTUNIDADES (MIN:MAX) } & \multicolumn{2}{|r|}{ DEBILIDADES - AMENAZAS (MIN:MAX) } \\
\hline D01 & $\begin{array}{l}\text { Educación ambiental mediante la partici- } \\
\text { pación comunitaria. }\end{array}$ & DA1 & $\begin{array}{l}\text { Recuperación, aprovechamiento y comer- } \\
\text { cialización de los residuos. }\end{array}$ \\
\hline DO2 & $\begin{array}{l}\text { Diseño y operación de un centro de aco- } \\
\text { pio de residuos peligrosos y de residuos } \\
\text { aprovechables. }\end{array}$ & DA2 & $\begin{array}{l}\text { Análisis de las necesidades de talento hu- } \\
\text { mano, maquinaria y equipos. }\end{array}$ \\
\hline DO3 & $\begin{array}{l}\text { Crear y propiciar la separación en la } \\
\text { fuente. }\end{array}$ & DA3 & $\begin{array}{l}\text { Generar hábitos de separación en la fuente } \\
\text { en los generadores de residuos sólidos. }\end{array}$ \\
\hline \multirow[t]{2}{*}{ DO4 } & $\begin{array}{l}\text { Recuperación, aprovechamiento y comer- } \\
\text { cialización de los residuos. }\end{array}$ & DA4 & $\begin{array}{l}\text { Apoyo a procesos de aprovechamiento, re- } \\
\text { húso y comercialización }\end{array}$ \\
\hline & & DA5 & $\begin{array}{l}\text { Evitar prácticas de disposición final de re- } \\
\text { siduos sólidos ambientalmente inseguras, } \\
\text { mediante el fortalecimiento de recolección y } \\
\text { transporte de residuos. }\end{array}$ \\
\hline
\end{tabular}

Fuente: elaboración propia

\section{Planteamiento de Alternativas}

Se realizó una serie de programas para implementar un sistema de gestión de residuos y optimizar el manejo existente. Adicionalmente, para cada programa se creó una ficha de manejo que amplía la información sobre cada una de las alternativas como se muestra en la tabla 3. 
Tabla 3. Programas ambientales para el manejo de los residuos sólidos y subproductos [11].

\begin{tabular}{|c|c|c|c|c|}
\hline PROGRAMA & PROYECTOS & DESCRIPCIYN & OBJETIVOS & META \\
\hline \multirow{3}{*}{$\begin{array}{l}\text { Integración de } \\
\text { la comunidad en } \\
\text { el manejo y dis- } \\
\text { posición de los } \\
\text { residuos sólidos }\end{array}$} & $\begin{array}{l}\text { Conformación y } \\
\text { capacitación a } \\
\text { grupos de sepa- } \\
\text { ración y recolec- } \\
\text { ción. }\end{array}$ & $\begin{array}{l}\text { Apoyo por parte de la } \\
\text { gerencia general para } \\
\text { la conformación de } \\
\text { grupos para la separa- } \\
\text { ción y recolección de } \\
\text { los residuos. } \\
\end{array}$ & $\begin{array}{l}\text { Conformar grupos de re- } \\
\text { cuperadores legalmente } \\
\text { constituidos para el aprove- } \\
\text { chamiento y valorización de } \\
\text { residuos sólidos }\end{array}$ & $\begin{array}{l}\text { Conformar } \\
\text { legalmente } \\
\text { por lo menos } \\
\text { dos grupos } \\
\text { de separación } \\
\text { y recolección. } \\
\end{array}$ \\
\hline & \multirow[b]{2}{*}{$\begin{array}{l}\text { Educación ambi- } \\
\text { ental. }\end{array}$} & \multirow{2}{*}{\begin{tabular}{|l|} 
Realizar campañas en \\
la comunidad, con el fin \\
de promover técnicas \\
adecuadas para el \\
manejo de los residuos \\
sólidos en los compo- \\
nentes de la gestión \\
integral.
\end{tabular}} & $\begin{array}{l}\text { 1. Campañas de separación } \\
\text { en la fuente. }\end{array}$ & \multirow{2}{*}{$\begin{array}{l}\text { Realizar } \\
\text { campañas } \\
\text { de educación } \\
\text { ambiental por } \\
\text { lo menos al } \\
90 \% \text { de los } \\
\text { colabora- } \\
\text { dores. } \\
\end{array}$} \\
\hline & & & $\begin{array}{l}\text { 2. Mejorar el ambiente de } \\
\text { trabajo mediante el buen } \\
\text { manejo y disposición de los } \\
\text { residuos. }\end{array}$ & \\
\hline \multirow{3}{*}{$\begin{array}{l}\text { Reúso, aprove- } \\
\text { chamiento y co- } \\
\text { mercialización de } \\
\text { los residuos }\end{array}$} & \multirow{2}{*}{$\begin{array}{l}\text { Recambio o ven- } \\
\text { ta de residuos } \\
\text { aprovechables. }\end{array}$} & \multirow{3}{*}{$\begin{array}{l}\text { Realizar la comercial- } \\
\text { ización de los residuos } \\
\text { sólidos potencialmente } \\
\text { aprovechables y re- } \\
\text { cambio de especiales. } \\
\text { Igualmente, la elabo- } \\
\text { ración de compost para } \\
\text { el aprovechamiento de } \\
\text { los residuos orgánicos } \\
\text { generados así como de } \\
\text { los subproductos. }\end{array}$} & $\begin{array}{l}\text { 1. Disminuir la cantidad de } \\
\text { residuos sólidos, que se dis- } \\
\text { ponen en el relleno sanitario }\end{array}$ & \multirow{3}{*}{$\begin{array}{l}\text { Realizar apr- } \\
\text { ovechamiento } \\
\text { por lo menos } \\
\text { del } 50 \% \text { de } \\
\text { residuos gen- } \\
\text { erados. }\end{array}$} \\
\hline & & & $\begin{array}{l}\text { 2. Fomentar la cultura de la } \\
\text { no basura }\end{array}$ & \\
\hline & Compostaje & & $\begin{array}{l}\text { 3. Incrementar los ingresos } \\
\text { de los grupos dedicados al } \\
\text { aprovechamiento de residuos }\end{array}$ & \\
\hline \multirow{2}{*}{$\begin{array}{l}\text { Optimización en } \\
\text { los componentes } \\
\text { de recolección y } \\
\text { transporte }\end{array}$} & \multirow{2}{*}{$\begin{array}{l}\text { Mejorar el siste- } \\
\text { ma de transporte } \\
\text { de los residuos } \\
\text { hacia su centro } \\
\text { de acopio y des- } \\
\text { tino final. }\end{array}$} & \multirow{2}{*}{$\begin{array}{l}\text { Incrementar las rutas } \\
\text { de evacuación de los } \\
\text { residuos hacia la celda } \\
\text { y el relleno de seguri- } \\
\text { dad. }\end{array}$} & $\begin{array}{l}\text { 1. Mejorar reponer o adquirir } \\
\text { equipos para el manejo y } \\
\text { recolección. }\end{array}$ & \multirow{2}{*}{$\begin{array}{l}\text { Llegar a una } \\
\text { cobertura } \\
\text { del } 100 \% \text { en } \\
\text { cuanto a re- } \\
\text { colección y } \\
\text { transporte. } \\
\end{array}$} \\
\hline & & & $\begin{array}{l}\text { 2. Diseñar de rutas de re- } \\
\text { colección que cubra la totali- } \\
\text { dad de la plantación. }\end{array}$ & \\
\hline \multirow{2}{*}{$\begin{array}{l}\text { Optimización } \\
\text { del manejo y } \\
\text { tratamiento de los } \\
\text { residuos y sub- } \\
\text { productos }\end{array}$} & \multirow{2}{*}{$\begin{array}{l}\text { Aprovechamien- } \\
\text { to de los subpro- } \\
\text { ductos industria- } \\
\text { les. }\end{array}$} & \multirow{2}{*}{$\begin{array}{l}\text { Emplear los subproduc- } \\
\text { tos industriales dentro } \\
\text { del mismo proceso pro- } \\
\text { ductivo del Biodiesel. }\end{array}$} & $\begin{array}{l}\text { 1. Caracterizar los subpro- } \\
\text { ductos para conocer su com- } \\
\text { posición y determinar sus } \\
\text { propiedades. }\end{array}$ & \multirow{2}{*}{$\begin{array}{l}\text { Iniciar los } \\
\text { programas de } \\
\text { programas de } \\
\text { monitoreo y } \\
\text { seguimiento } \\
\text { de los sub- } \\
\text { productos } \\
\text { empleados. } \\
\end{array}$} \\
\hline & & & $\begin{array}{l}\text { 2. Determinar las mejores } \\
\text { alternativas de compostaje y } \\
\text { aprovechamiento de los sub- } \\
\text { productos. }\end{array}$ & \\
\hline $\begin{array}{l}\text { Mejoramiento de } \\
\text { la gestión de los } \\
\text { residuos sólidos y } \\
\text { subproductos }\end{array}$ & $\begin{array}{l}\text { Capacitación al } \\
\text { personal encar- } \\
\text { gado del manejo } \\
\text { recolección y } \\
\text { disposición final } \\
\text { de residuos sóli- } \\
\text { dos. }\end{array}$ & $\begin{array}{l}\text { Capacitar al personal } \\
\text { involucrado en la ca- } \\
\text { dena de generación, } \\
\text { manejo y disposición } \\
\text { de los residuos y sub- } \\
\text { productos. }\end{array}$ & $\begin{array}{l}\text { Capacitar al personal en- } \\
\text { cargado de la recolección, } \\
\text { manejo y transporte de los } \\
\text { residuos sólidos y subpro- } \\
\text { ductos industriales con el fin } \\
\text { de prestar un servicio efici- } \\
\text { ente y seguro en términos de } \\
\text { condiciones óptimas de salud } \\
\text { ocupacional. }\end{array}$ & $\begin{array}{l}\text { Capacitar } \\
\text { al } 100 \% \text { de } \\
\text { los colabora- } \\
\text { dores de la } \\
\text { cadena de } \\
\text { residuos y } \\
\text { subproductos. }\end{array}$ \\
\hline
\end{tabular}




\section{Alternativa 1. El compostaje.}

Es un proceso de degradación realizado por microorganismos, los cuales en presencia de la humedad, con temperatura adecuada y bajo condiciones aeróbicas, utilizan los nutrientes que están contenidos en el material por trasformar hasta obtener un producto homogéneo denominado compost, libre de patógenos y estabilizado físico-químicamente, este compost se aplicaría en los lotes de la plantación, minimizando el uso de agroquímicos como fertilizantes (ver Tabla 4).

Esta es una propuesta para la cual se han presentado dos alternativas, las cuales se encuentran en estudio de factibilidad técnica y económica, las cuales en cálculos preliminares estimados, oscilan entre US $\$ 534.000$ y US $\$ 667.000$; sin embargo, a la fecha no se cuenta con una propuesta final de los proveedores, (BIOTECH, AV COMPOSTING Ltd.).

\section{Beneficios de la alternativa 1.}

- Todos los desechos orgánicos se utilizan en proporciones específicas en el proceso de compostaje.

- El compost puede ser aprovechado en los cultivos de inmediato y en su totalidad.

- Permite el manejo racional de volúmenes elevados.

- Mejora estructura y propiedades físicas del suelo.

- Se devuelve a campo un producto de bajo costo, obtenido en tiempo inferior a 40 días, biodegradado, estabilizado que aporta nutrientes a la planta.

- Se incrementa la productividad de las plantaciones.

- Posibilita la sustitución parcial de la fertilización química por orgánica, de manera gradual, para acondicionar suelos y mejorar calidad de los frutos.

- Es un proceso acorde con las normas de calidad ISO 14000 y las exigencias de manejo ambiental.

\section{Desventajas de la alternativa 1.}

- Manejo ambientalmente responsable pero no ambientalmente sostenible.

- Disponibilidad de 5 o 6 hectáreas de terreno para el proceso de compostaje.

- Costos de mantenimiento, personal y maquinaria.

- Generación leve de olores.

- Costo de implementación del sistema de compostaje.

Tabla 4. Análisis comparativo entre la descomposición tradicional y el compostaje.

\begin{tabular}{||l|c|c||}
\hline \multicolumn{1}{|c|}{ VARIABLE ANELISIS } & TRADICIONAL & COMPOSTAJE \\
\hline Tiempo de degradación del Raquis & 240 Días & 40 Días \\
\hline Tipo de proceso & No Controlado & Controlado \\
\hline Calidad del producto & Menor & Mayor \\
\hline Aprovechamiento residuos & Parcial & Total \\
\hline Facilidad de transporte a campo & Menor & Mayor \\
\hline Generación de residuos orgánicos ha/año & 5 ton/Raquis & 7 ton/Compost \\
\hline Aplicación actual/ha & $50-100$ ton Raquis & $70-140$ ton Compost \\
\hline
\end{tabular}

Fuente: Elaboración propia 


\section{Alternativa 2. Disposición por un tercero (gestor de residuos).}

Se consultaron diferentes firmas que ofrecen los servicios de manejo, transporte, tratamiento y disposición final de los residuos generados por la planta, aceptadas por la Autoridad Ambiental; los valores ofertados están de acuerdo con la cantidad de residuos, vigencia del contrato y características particulares del mismo, (ver Tabla 5).

\section{Beneficios de la alternativa 2.}

- No se generan pasivos ambientales, ni residuos, ni emisiones durante el proceso.

- No se requiere mantenimiento, ni acondicionamiento de áreas dentro de las instalaciones de la empresa.

- El transporte del material se realiza conforme con la normatividad legal vigente.

- Se garantiza la correcta disposición de los residuos de acuerdo a su tipo y con la infraestructura adecuada, para su co-procesamiento, disposición final, incineración, encapsulamiento.

- Asesoría técnica ambiental permanente.

\section{Desventajas de la alternativa 2.}

- Manejo ambientalmente responsable pero no ambientalmente sostenible.

- Elevados costos de disposición sin inversiones en la empresa.

- Tercerización del riesgo y la responsabilidad del manejo de los residuos o subproductos.

Tabla 5. Análisis de costos para la alternativa 2

\begin{tabular}{|c|c|c|c|c|}
\hline EMPRESA & $\begin{array}{c}\text { PRECIO UNITARIO } \\
\text { (US\$/Ton) }\end{array}$ & $\begin{array}{c}\text { VALOR ANUAL } \\
\text { ESTIMADO US\$ }\end{array}$ & $\begin{array}{c}\text { VALORES } \\
\text { ADICIONALES }\end{array}$ & $\begin{array}{c}\text { TIEMPO DE } \\
\text { CONTRATO }\end{array}$ \\
\hline GEREP S.A. E.S.P. & 500 & 1.022 .000 & Cargue: US\$10/ton & 2 AÑOS \\
\hline CONSORCIO SAAM & 435 & 819.000 & $\begin{array}{c}\text { Transporte: US\$2.200/ } \\
\text { mula }\end{array}$ & sin período \\
\hline ECOEFICIENCIA & 700 & 1.340 .000 & Transporte & sin período \\
\hline
\end{tabular}

Fuente: Elaboración propia

\section{Alternativa 3. Almacenamiento interno de residuos previo aplicación en campo (práctica aprobada por el ICA).}

Se plantea la construcción de un centro de almacenamiento de los residuos o subproductos como raquis, tierras filtrantes, cenizas, lodos, con el fin de acopiarlos previa a su aplicación en los meses de verano en los lotes de campo, (ver Tabla 6).

\section{Beneficios de la alternativa 3.}

- El producto de la descomposición puede ser aprovechado en los cultivos de inmediato y en su totalidad.

- Permite el manejo racional de volúmenes elevados.

- Mejora estructura y propiedades físicas del suelo.

- Se devuelve a campo un producto de bajo costo.

- Se incrementa la productividad de las plantaciones.

- Posibilidad de sustituir parcialmente la fertilización química por orgánica, de manera gradual, para acondicionar suelos y mejorar calidad de los frutos. 
Desventajas de la alternativa 3.

- Generación de vectores como olores ofensivos y moscas

- Costos de mantenimiento, maquinaria y transporte.

- Construcción de la infraestructura necesaria para el almacenamiento del material.

- No se puede emplear todo el material orgánico presente en la plantación.

Tabla 6. Análisis de Costos de la alternativa 3

\begin{tabular}{|c|c|c|}
\hline EMPRESA & ESPECIFICACIONES & $\begin{array}{c}\text { VALOR ANUAL ESTIMADO } \\
\text { US\$ }\end{array}$ \\
\hline KUBRIR LTDA & $\begin{array}{l}\text { Postes en concreto } \\
\text { Polietileno para canal y cubierta } \\
\text { Polisombra } \\
\text { Cable acerado y varilla de anclaje } \\
\text { Zapatas y tensores } \\
\text { Transporte } \\
\text { Piso en concreto } \\
\text { Herramientas y materiales } \\
\text { Diseño y trazado } \\
\text { Placas en concreto }\end{array}$ & 487.000 \\
\hline CPE & $\begin{array}{l}\text { Postes en concreto } \\
\text { Polietileno para canal y cubierta } \\
\text { Polisombra } \\
\text { Cable acerado y varilla de anclaje } \\
\text { Zapatas y tensores } \\
\text { Transporte } \\
\text { Piso en concreto } \\
\text { Herramientas y materiales } \\
\text { Diseño y trazado } \\
\text { Placas en concreto }\end{array}$ & 534.000 \\
\hline $\begin{array}{l}\text { BIOAGRICOLA DEL } \\
\text { LLANO }\end{array}$ & $\begin{array}{l}\text { Diseño centro de almacenamiento de re- } \\
\text { siduos o subproductos }\end{array}$ & 4.000 \\
\hline
\end{tabular}

Fuente: Elaboración propia 


\section{Conclusiones}

Como parte de la evaluación del PMA se pudo evidenciar la necesidad de una evaluación de impactos reales para todas las etapas de producción, para lo cual se requiere una metodología que se ajusta a las características propias de este negocio. Las actividades propias de la plantación y las de la construcción de la planta de biodiesel requieren el desmonte y el acondicionamiento de los suelos, además con la incorporación de químicos se altera las propiedades químicas y físicas del suelo. La aplicación de las técnicas adecuadas y a tiempo ayudan a la remediación de este recurso, además evitara la degradación del suelo haciendo que este se recupere en menor tiempo y las implicaciones ambientales negativas sean tenues [12].

Las áreas que abarcan los procesos son susceptibles a la contaminación del aire debido a la emisión de partículas sólidas y gaseosas que se generan en su mayoría por los procesos que se llevan a cabo en la planta extractora de aceite y la operación de la planta de biodiesel [13]. Los mayores impactos negativos para la calidad del aire son los que provienen de la extracción del aceite de palma, debido a este diagnóstico, es más fácil ejercer un control en la fuente con diferentes técnicas o empleo de tecnología avanzada que ayude a minimizar estos efectos. Se recomienda la adquisición de calderas modernas para reemplazar las antiguas, aunque las nuevas calderas también presentaran niveles de contaminación que superan los límites de la Norma se hace necesario entonces instalar un sistema de control de emisiones [14].

En el manejo de residuos, que es el tema que impulsó el desarrollo de este proyecto, presenta el inconveniente de tener residuos que por la localización geográfica no pueden ser dispuestos con facilidad, además de los altos costos por su transporte y manejo. Esto pone en la balanza el costo del manejo de los residuos por un tercero, versus la creación de una planta compostera en donde se obtendría una recuperación de la inversión, seguida de una considerable reducción en los costos de producción de la empresa, todo esto encaminado a que la compostera ahorraría costos de manejo y tratamiento del subproducto por un tercero y se haría internamente para aumentar de igual forma las propiedades fertilizantes del suelo. A esto se le suma el ahorro de las sanciones gubernamentales. Finalmente, se deberá incorporar en el PMA el Plan de Emergencias donde se indican los procedimientos por implementar y los aspectos en el caso de presentarse alguna emergencia ambiental [15].

\section{Agradecimiento}

Este documento se realizó con la colaboración de Aceites Manuelita S.A, gracias a su información y a todo el personal que brindaron datos de vital importancia para el desarrollo de esta investigación.

\section{Referencias Bibliográficas}

[1] Beltrán S. y Zamora A. Alcaldía Municipal de San Carlos de Guaroa Meta (2000).

[2] Ahmad, Sarwani Hj Adni, Chang, Ah Know Ho, Cheng Tuck \& Teo, Cheng Hai (1999).

[3] Chan, Kook Weng, Ma, Ah Ngan, Ariffin, Darus \& Jalani, B.Sukaimi (1999).

[4] PORIM. Chávez López, E. (2009). Palma africana entre el cielo y el infierno. Revista Ambiental. Catorce 6. No. 29. Ed. Corporación Bioparque. Bogotá D. C. Disponible: http://www.catorce6.com/ la-revista/

[5] Enríquez Bernal, L. M. (2009). Estrategia para la implementación de la Norma ISO 14001 en empresas productoras de palma de aceite de la zona oriental colombiana. Tesis de Maestría en Gestión Ambiental. Facultad de Estudios Ambientales y Rurales, Pontificia Universidad Javeriana, Bogotá D. C. 
[6] CPA Ltda. (2008). Plan de Manejo Ambiental para Aceites Manuelita S. A.

[7] Arenas Ferro, J. (2010). Brevísima narración sobre los bioagro- combustibles. Gestión y Ambiente. Volumen 13, No. 1. ISSN 0124-177X.

[8] Conesa Fernández-Vítora, V. (2009). Guía Metodológica para la evaluación del impacto ambiental: (4ta Ed.). Madrid: Ediciones Mundi-Prensa.. ISBN 978-84-8476-384-0.

[9] Infante Villareal, A. (2007). Perspectivas de la situación energética mundial. Las oportunidades para Colombia. Revista de Ingeniería, No. 25, ISSN 0121-4993.

[10] Liew, Hsien Ta \& Tan, Bee Wah. (1999). Ruta para el sistema de manejo ambiental“Nuestra responsabilidad ambiental"(Experiencia en plantaciones de sime Darby). 1999 PIPOC PORIM.

[11] Maldonado Romero, M. (2010). Evaluación del PMA en una planta de producción de biodiesel. Tra- bajo de grado para optar al título de Ingeniera Ambiental y Sanitaria. Programa de Ingeniería Ambiental y Sanitaria, Facultad de Ingeniería, Universidad de La Salle, Bogotá D.C.

[12] Espinosa, G. (2001). Fundamentos de evaluación de Impacto Ambiental. Banco Interamericano de Desarrollo - BID, Santiago de Chile, Chile: Centro de Estudios para el Desarrollo - CED.

[13] FEDEPALMA (2009). Biodiésel de palma: un proyecto de largo de largo aliento convertido en realidad. Palmas. Vol. 29 No. 1. ISSN 0121-2923.

[14] Heson, I. E. (1995). Impactos ambientales de las plantaciones de palma de aceite en Malasia. Palmas. Vol. 16, No. 4, p.49-66. ISSN 0121-2923.

[15] Reinosa Pulido, D. C. (2009). Costos ambientales en el proceso de extracción del aceite de palma. Estudio de caso. Revista Venezolana de Gerencia, Vol. 14, No. 46, pp. 228-247 Universidad del Zulia, Venezuela. ISSN 1315-9984.

[16] Evaluación del impacto ambiental de plantaciones de palma de aceite en bosques secundarios. Expe- riencias del Golden Hope. 1999 PIPOC PORIM.

[17] International Palm Oil Congress Emergin Technologies and Opportunities in the next Millennium (February 1-6: Kuala Lumpur, Malaysia. Proceedings (Chemistry and Technology)). Kuala Lumpur: PORIM.

[18] CORPORINOQUIA (2000). Documento técnico: Plan de Manejo Ambiental (PMA) establecido para la plantación y planta extractora. Resolución 473 del 11 de Agosto de 2000.

[19] Documento Técnico: Ordenamiento Territorial de Guaroa.

[20] Evaluación del comportamiento ambiental de la industria de la palma de aceite en Malasia. PIPOC PORIM International Palm Oil Congress Emergin Technologies and Opportunities in the next Millennium (February 1-6 : Kuala Lumpur, Malaysia. Proceedings- (Chemistry and Technology). (1999) Kuala Lumpur.

[21] Garmendia Salvador, A. (2005). Evaluación de Impacto Ambiental. Ed. Pearson Alhambra. ISBN: 8420543985.

[22] Gómez Orea, D. (2002). Evaluación de Impacto Ambiental un instrumento preventivo para la gestión ambiental. Ediciones Mundi-Prensa, Madrid, 2ª Edición. ISBN 84-8476- 084-7.

[23] International Palm Oil Congress Emergin Technologies and Opportunities in the next Millen- nium (February 1-6 : Kuala Lumpur, Malaysia. Proceedings (Chemistry and Technology)). Kuala Lumpur: PORIM, 1999.

[24] República de Colombia, Ministerio de Medio Ambiente, FEDEPALMA (2002). Guía Ambiental para el subsector de la Agroindustria de la Palma de Aceite. ISBN 9789589649473.

[25] Yañez, E. (2009). Biocombustibles, medio ambiente, tecnología y seguridad alimentaria. Palmas Vol. 30, No. 1, p.71-91. ISSN 0121-2923. 